\title{
NORMAL FAMILIES OF MEROMORPHIC MAPPINGS OF SEVERAL COMPLEX VARIABLES INTO $\mathbf{P}^{N}(\mathrm{C})$
}

\author{
PHAM NGOC MAI, DO DUC THAI AND \\ PHAM NGUYEN THU TRANG
}

Dedicated to Professor Hirotaka Fujimoto at his retirement

\begin{abstract}
The first aim in this article is to give some sufficient conditions for a family of meromorphic mappings of a domain $D$ in $\mathbf{C}^{n}$ into $\mathbf{P}^{N}(\mathbf{C})$ omitting hypersurfaces to be meromorphically normal. Our result is a generalization of the results of Fujimoto and Tu. The second aim is to investigate extending holomorphic mappings into the compact complex space from the viewpoint of the theory of meromorphically normal families of meromorphic mappings.
\end{abstract}

\section{$\S 1$. Introduction}

Classically, a family $\mathcal{F}$ of holomorphic functions on a domain $D \subset \mathbf{C}$ is said to be (holomorphically) normal if every sequence in $\mathcal{F}$ contains a subsequence which converges uniformly on all the compact subsets of $D$.

In 1957 Lehto and Virtanen [LeVi] introduced the concept of normal meromorphic functions in connection with the study of boundary behaviour of meromorphic functions of one complex variable. Since then normal holomorphic maps has been studied intensively, resulting in an extensive development in the single complex variable context and in generalizations to several complex variables settings (see [Za], [JK1], [JK2], [AK] and the references cited in [Za] and [JK2]).

The first ideas and results on normal families of meromorphic mappings of several complex variables were introduced by Rutishauser [Rut] and Stoll $[\mathrm{S}]$.

The notion of a meromorphically normal family into the $N$-dimensional complex projective space is introduced by $\mathrm{H}$. Fujimoto [Fu2]. He also gave

Received February 25, 2003.

1991 Mathematics Subject Classification: 32A10, 32C10, 32H20, 32A17, 54C20, 54D35, 54C05. 
some sufficient conditions for a family of meromorphic mappings of a domain $D$ in $\mathbf{C}^{n}$ into $\mathbf{P}^{N}(\mathbf{C})$ to be meromorphically normal. As a weaker version of the concept of meromorphic normality, H. Fujimoto [Fu2] introduced the concept of quasi-normality. Recently, Z. Tu [Tu2] considered meromorphically normal families of meromorphic mappings of a domain $D$ in $\mathbf{C}^{n}$ into $\mathbf{P}^{N}(\mathbf{C})$ omitting hyperplanes. See 3.1 for the actual definition of these concepts.

The first aim in this article is to give some sufficient conditions for a family of meromorphic mappings of a domain $D$ in $\mathbf{C}^{n}$ into $\mathbf{P}^{N}(\mathbf{C})$ omitting hypersurfaces to be meromorphically normal or quasi-normal. These results are generalizations of the above Fujimoto's and Tu's results.

The second aim of this article is to investigate extending holomorphic mappings into compact complex spaces from the viewpoint of the theory of meromorphically normal families of meromorphic mappings. In order to state our main result, we need some preliminary.

First, for hypersurfaces $H_{i}(1 \leq i \leq q)$ of $\mathbf{P}^{N}(\mathbf{C})$ with $q \geq N+1$, let $Q_{i}(1 \leq i \leq q)$ be their defining polynomials, i.e., the homogeneous polynomials without multiple factors such that

$$
H_{i}=\left\{z=\left(z_{0}: z_{1}: \cdots: z_{N}\right): Q_{i}(z)=0\right\}
$$

Here and below, throughout the article, we only consider homogeneous polynomials $Q(z)=\sum a_{\nu} z^{\nu}$ normalized so that $\sum\left|a_{\nu}\right|^{2}=1$. Now we define

$$
\begin{aligned}
D\left(H_{1}, \ldots, H_{q}\right) & =D\left(Q_{1}, \ldots, Q_{q}\right) \\
& =\prod_{1 \leq i_{1}<i_{2}<\cdots<i_{N+1} \leq q} \inf _{|z|=1}\left(\left|Q_{i_{1}}(z)\right|^{2}+\cdots+\left|Q_{i_{N+1}}(z)\right|^{2}\right)
\end{aligned}
$$

where $\|z\|=\left(\sum\left|z_{j}\right|^{2}\right)^{1 / 2}$.

Next, let $\Lambda^{d}(S)$ denote the real $d$-dimensional Hausdorff measure of $S \subset \mathbf{C}^{n}$. For a formal Z-linear combination $X=\sum_{i \in I} n_{i} X_{i}$ of analytic subsets $X_{i} \subset \mathbf{C}^{n}$ and for a subset $E \subset \mathbf{C}^{n}$, we call $\sum_{i \in I} \Lambda^{d}\left(X_{i} \cap E\right)$ (resp. $\left.\sum_{i \in I} n_{i} \Lambda^{d}\left(X_{i} \cap E\right)\right)$, the $d$-dimensional Lebesgue area of $X \cap E$ regardless of multiplicities (resp. with counting multiplicities).

Now we can state our main results. 
THEOREM A. Let $\mathcal{F}$ be a family of meromorphic mappings of a domain $D$ in $\mathbf{C}^{n}$ into $\mathbf{P}^{N}(\mathbf{C})$. Suppose that for each $f \in \mathcal{F}$, there exist $q \geq 2 N+1$ hypersurfaces $H_{1}(f), H_{2}(f), \ldots, H_{q}(f)$ in $\mathbf{P}^{N}(\mathbf{C})$ with

$$
\begin{gathered}
\inf \left\{D\left(H_{1}(f), \ldots, H_{q}(f)\right) ; f \in \mathcal{F}\right\}>0 \quad \text { and } \\
f(D) \not \subset H_{i}(f) \quad(1 \leq i \leq N+1),
\end{gathered}
$$

where $q$ is independent of $f$, but the hypersurfaces $H_{i}(f)$ may depend on $f$, such that the following two conditions are satisfied:

i) For any fixed compact subset $K$ of $D$, the $2(n-1)$-dimensional Lebesgue areas of $f^{-1}\left(H_{i}(f)\right) \cap K(1 \leq i \leq N+1)$ with counting multiplicities for all $f$ in $\mathcal{F}$ are bounded above.

ii) There exists a closed subset $S$ of $D$ with $\Lambda^{2 n-1}(S)=0$ such that for any fixed compact subset $K$ of $D-S$, the $2(n-1)$-dimensional Lebesgue areas of $f^{-1}\left(H_{i}(f)\right) \cap K(N+2 \leq i \leq q)$ with counting multiplicities for all $f$ in $\mathcal{F}$ are bounded above.

Then $\mathcal{F}$ is a meromorphically normal family on $D$.

THEOREM B. Let $\mathcal{F}$ be a family of meromorphic mappings of a domain $D$ in $\mathbf{C}^{n}$ into $\mathbf{P}^{N}(\mathbf{C})$. Suppose that for each $f \in \mathcal{F}$, there exist $q \geq 2 N+1$ hypersurfaces $H_{1}(f), H_{2}(f), \ldots, H_{q}(f)$ in $\mathbf{P}^{N}(\mathbf{C})$ with

$$
\inf \left\{D\left(H_{1}(f), \ldots, H_{q}(f)\right) ; f \in \mathcal{F}\right\}>0,
$$

where $q$ is independent of $f$, but the hypersurfaces $H_{i}(f)$ may depend on $f$, such that for any fixed compact subset $K$ of $D$, the $2(n-1)$-dimensional Lebesgue areas of $f^{-1}\left(H_{i}(f)\right) \cap K(1 \leq i \leq q)$ with counting multiplicities for all $f \in \mathcal{F}$ are bounded above. Then $\mathcal{F}$ is a quasi-normal family on $D$.

Theorem C. Let $\Omega$ be a domain in $\mathbf{C}^{n}$ and $S \subset \Omega$ an analytic subset of codimension 1, whose singularities are normal crossings. Let $M$ be a compact complex space. Let $f \in \operatorname{Hol}(\Omega-S, M)$. Suppose that there exist $q$ hypersurfaces $H_{1}, \ldots, H_{q}$ in $M$ and fixed positive integers $m_{1}, \ldots, m_{q}\left(m_{i} \leq\right.$ $\infty, i=1, \ldots, q)$ such that the family $\left\{\left(H_{i}, m_{i}\right)\right\}_{i=1}^{q}$ has the D-property (cf. Definition 4.3) and $f$ intersects $H_{i}$ with multiplicity at least $m_{i}$ for each $1 \leq i \leq q$. Then $f$ extends to a holomorphic mapping $f^{*}: \Omega \rightarrow M$.

Acknowledgement. We would like to express our gratitude for the referee. His/her valuable comments made on the first version of this paper led to significant improvements in the paper. 


\section{$\S 2 . \quad$ Notations}

2.1. Let $A$ be a non-empty open subset of a domain $D$ in $\mathbf{C}^{n}$ such that $S=D-A$ is an analytic set in $D$. Let $f: A \rightarrow \mathbf{P}^{N}(\mathbf{C})$ be a holomorphic mapping. Let $U$ be a non-empty connected open subset of D. A holomorphic mapping $\tilde{f} \not \equiv 0$ from $U$ into $\mathbf{C}^{N+1}$ is said to be a representation of $f$ on $U$ if $f(z)=\rho(\tilde{f}(z))$ for all $z \in U \cap A-\tilde{f}^{-1}(0)$, where $\rho: \mathbf{C}^{N+1}-\{0\} \rightarrow \mathbf{P}^{N}(\mathbf{C})$ is the standard projective mapping. A holomorphic mapping $f: A \rightarrow \mathbf{P}^{N}(\mathbf{C})$ is said to be a meromorphic mapping from $D$ into $\mathbf{P}^{N}(\mathbf{C})$ if and only if for any $z \in D$, there exists a representation of $f$ on some neighborhood of $z$ in $D$.

2.2. Let $D$ be a domain in $\mathbf{C}^{n}$ and $f$ a not identically zero holomorphic function on $D$. For a point $a=\left(a_{1}, a_{2}, \ldots, a_{n}\right) \in D$ we expand $f$ as a compactly convergent series

$$
f\left(u_{1}+a_{1}, \ldots, u_{n}+a_{n}\right)=\sum_{m=0}^{\infty} P_{m}\left(u_{1}, \ldots, u_{n}\right)
$$

on a neighborhood of $a$, where $P_{m}$ is either identically zero or a homogeneous polynomial of degree $m$. The number

$$
\nu_{f}(a):=\min \left\{m ; P_{m}(u) \not \equiv 0\right\}
$$

is said to be the zero multiplicity of $f$ at $a$. By definition, a divisor on $D$ is an integer-valued function $\nu$ on $D$ such that for every $a \in D$ there are holomorphic functions $g(z)(\not \equiv 0)$ and $h(z)(\not \equiv 0)$ on a neighborhood $U$ of $a$ with $\nu(z)=\nu_{g}(z)-\nu_{h}(z)$ on $U$. We define the $\operatorname{support} \operatorname{supp} \nu$ of the divisor $\nu$ on $D$ by

$$
\operatorname{supp} \nu:=\overline{\{z \in D: \nu(z) \neq 0\}} .
$$

We denote $\mathcal{D}^{+}(D)=\{\nu$ : a non-negative divisor on $D\}$.

2.3. Let $f$ be a meromorphic mapping of a domain $D$ in $\mathbf{C}^{n}$ into $\mathbf{P}^{N}(\mathbf{C})$. Then for any $a \in D, f$ always has a representation

$$
\tilde{f}(z)=\left(f_{0}(z): f_{1}(z): \cdots: f_{N}(z)\right)
$$

on some neighborhood $U$ of $a$ with fixed homogeneous coordinates $\left(w_{0}\right.$ : $\left.w_{1}: \cdots: w_{N}\right)$ on $\mathbf{P}^{N}(\mathbf{C})$ and holomorphic functions $f_{i}(z)(0 \leq i \leq N)$ on $U$, where we can choose them so as to satisfy the condition

$$
\operatorname{codim}\left\{f_{0}(z)=f_{1}(z)=\cdots=f_{N}(z)=0\right\} \geq 2 .
$$


A representation of $f$ satisfying this condition is refered to as an admissible representation of $f$ on $U$ in the following sections.

For a meromorphic mapping $f$ into $\mathbf{P}^{N}(\mathbf{C})$ we denote by $I(f)$ the set of all points of indetermination of $f$, which is given by the condition

$$
I(f) \cap U=\left\{z \in U: f_{0}(z)=f_{1}(z)=\cdots=f_{N}(z)=0\right\}
$$

if $f$ has an admissible representation $f=\left(f_{0}: f_{1}: \cdots: f_{N}\right)$ on an open subset $U$ of $D$. So, we have $\operatorname{codim} I(f) \geq 2$.

2.4. Take a hypersurface $H$ in $\mathbf{P}^{N}(\mathbf{C})$ defined by

$$
H:=\left\{\left(z_{0}: z_{1}: \cdots: z_{N}\right) \in \mathbf{P}^{N}(\mathbf{C}): Q^{d}\left(z_{0}, z_{1}, \ldots, z_{N}\right)=0\right\}
$$

where $Q^{d}$ is a homogeneous polynomial of degree $d$ on $\mathbf{C}^{N+1}$.

Let $D$ be a domain in $\mathbf{C}^{n}$. For any $a \in D$, taking an admissible representation $f=\left(f_{0}: f_{1}: \cdots: f_{N}\right)$ on a neighborhood $U$ of $a$, we consider a holomorphic function

$$
F:=Q^{d}\left(f_{0}, f_{1}, \ldots, f_{N}\right)
$$

Then, the divisor $\nu(f, H)(z):=\nu_{F}(z)(z \in U)$ is determined independently of a choice of admissible representations and hence is well-defined on the totality of $D$.

2.5. In 2.4 , we defined the divisor $\nu(f, H)(z):=\nu_{F}(z)(z \in U)$. Obviously, $\operatorname{supp} \nu(f, H)$ is either empty or a pure $(n-1)$-dimensional analytic set in $D$ if $f(D) \not \subset H$ (i.e., $F(z) \not \equiv 0$ on $U)$. We define $\nu(f, H)=\infty$ on $D$ and $\operatorname{supp} \nu(f, H)=D$ if $f(D) \subset H$. Sometimes we identify $f^{-1}(H)$ with the divisor $\nu(f, H)$ on $D$. Rewrite $\nu(f, H)$ as the formal sum $\nu(f, H)=$ $\sum_{i \in I} n_{i} X_{i}$, where $X_{i}$ are the irreducible components of $\operatorname{supp} \nu(f, H)$ and $n_{i}$ are the constant $\nu(f, H)(z)$ on $X_{i} \cap \operatorname{Reg}(\operatorname{supp} \nu(f, H))$, where $\operatorname{Reg}()$ denotes the set of all the regular points.

We say that a meromorphic mapping $f$ intersects $H$ with multiplicity at least $m$ on $D$ if $f(D) \not \subset H, f(D) \cap H \neq \emptyset$, and $\nu(f, H)(z) \geq m$ for all $z \in \operatorname{supp} \nu(f, H)$ and that $f$ intersects $H$ with multiplicity $\infty$ on $D$ if $f(D) \subset H$ or $f(D) \cap H=\emptyset$.

2.6. For each $x \in \mathbf{C}^{n}$ and $R>0$, we set $B(x, R)=\left\{z \in \mathbf{C}^{n}:\|z-x\|<\right.$ $R\}$ and $B(0, R)=B(R)$. 


\section{§3. Criterions for meromorphically normal families}

First of all, we recall some definitions.

Definition 3.1. Let $D$ be a domain in $\mathbf{C}^{n}$.

i) (see $[\mathrm{AK}]$ ) Let $\mathcal{F}$ be a family of holomorphic mappings of $D$ into a complex manifold $M . \mathcal{F}$ is said to be a holomorphically normal family on $D$ if any sequence in $\mathcal{F}$ contains a subsequence which converges uniformly on compact subsets of $D$ to a holomorphic mapping of $D$ into $M$.

ii) (see $[\mathrm{Fu} 2]$ ) A sequence $\left\{f^{(p)}(z)\right\}$ of meromorphic mappings from $D$ into $\mathbf{P}^{N}(\mathbf{C})$ is said to meromorphically converge on $D$ to a meromorphic mapping $f(z)$ if and only if, for any $z \in D$, each $f^{(p)}(z)$ has an admissible representation

$$
\tilde{f}^{(p)}=\left(f_{0}^{(p)}: f_{1}^{(p)}: \cdots: f_{N}^{(p)}\right)
$$

on some fixed neighborhood $U$ of $z$ such that $\left\{f_{i}^{(p)}(z)\right\}_{p=1}^{\infty}$ converges uniformly on compact subsets of $U$ to a holomorphic function $f_{i}(0 \leq i \leq N)$ on $U$ with the property that $\tilde{f}=\left(f_{0}: f_{1}: \cdots: f_{N}\right)$ is a representation of $f$ on $U$, where $f_{i_{0}} \neq \equiv 0$ on $U$ for some $i_{0}$.

iii) (see [Fu2]) Let $\mathcal{F}$ be a family of meromorphic mappings of $D$ into $\mathbf{P}^{N}(\mathbf{C}) . \quad \mathcal{F}$ is said to be a meromorphically normal family on $D$ if any sequence in $\mathcal{F}$ has a meromorphically convergent subsequence on $D$.

iv) (see $[\mathrm{Fu} 2]$ ) A sequence $\left\{f^{(p)}\right\}$ of meromorphic mappings from $D$ into $\mathbf{P}^{N}(\mathbf{C})$ is said to be quasi-regular on $D$ if and only if any $z \in D$ has a neighborhood $U$ with the property that $\left\{f^{(p)}\right\}$ converges compactly on $U$ outside a nowhere dense analytic subset $S$ of $U$, i.e., for any domain $G \Subset U-S$, there is some $p_{0}$ such that $I\left(f^{(p)}\right) \cap G=\emptyset\left(p \geq p_{0}\right)$ and $\left\{\left.f^{(p)}\right|_{G}, p \geq p_{0}\right\}$ converges uniformly on $G$ to a holomorphic mapping of $G$ into $\mathbf{P}^{N}(\mathbf{C})$.

Obviously a meromorphically convergent sequence on $D$ is always quasiregular sequence on $D$. But a quasi-regular sequence on $D$ need not imply meromorphic convergence on $D$.

v) (see [Fu2]) Let $\mathcal{F}$ be a family of meromorphic mappings of $D$ into $\mathbf{P}^{N}(\mathbf{C}) . \mathcal{F}$ is said to be a quasi-normal family on $D$ if any sequence in $\mathcal{F}$ has a subsequence so as to be quasi-regular on $D$.

vi) (see $[\mathrm{S}]$ ) Let $\left\{\nu_{i}\right\}_{i \in I}$ be a directed set of non-negative divisors on $D$. It is said to converge to a non-negative divisor $\nu$ on $D$ if and only if any $a \in D$ has a neighborhood $U$ such that, for suitable holomorphic functions 
$h_{i}(\not \equiv 0)$ and $h(\not \equiv 0)$ on $U, \nu_{i}=\nu_{h_{i}}, \nu=\nu_{h}$ and $\left\{h_{i}\right\}_{i \in I}$ converges compactly to $h$ on $U$.

Lemma 3.2. ([S, Theorem 4.10]) If a sequence $\left\{\nu_{i}\right\}$ converges to $\nu$ in $\mathcal{D}^{+}(B(R))$, then $\left\{\operatorname{supp} \nu_{i}\right\}$ converges to $\operatorname{supp} \nu$ in the sense that $\operatorname{supp} \nu$ coincides with the set of all $z$ such that every neighborhood $U$ of $z$ intersects $\operatorname{supp} \nu_{i}$ for all but finitely many $i$ and, simultaneously, with the set of all $z$ such that every $U$ intersects $\operatorname{supp} \nu_{i}$ for infinitely many $i$.

Lemma 3.3. ([S, Theorem 2.24]) A sequence $\left\{\nu_{i}\right\}$ of non-negative divisors on a domain $D$ in $\mathbf{C}^{n}$ is normal in the sense of the convergence of divisors on $D$ if and only if the $2(n-1)$-dimensional Lebesgue areas of $\nu_{i} \cap E(i \geq 1)$ with counting multiplicities are bounded above for any fixed compact set $E$ of $D$.

Lemma 3.4. Let $\left\{f^{(p)}\right\}$ be a sequence of meromorphic mappings of a domain $D$ in $\mathbf{C}^{n}$ into $\mathbf{P}^{N}(\mathbf{C})$ and let $S$ be a closed subset of $D$ with $\Lambda^{2 n-1}(S)=0$. Suppose that $\left\{f^{(p)}\right\}$ meromorphically converges on $D-S$ to a meromorphic mapping $f$ of $D-S$ into $\mathbf{P}^{N}(\mathbf{C})$. If there exists a hypersurface $H$ in $\mathbf{P}^{N}(\mathbf{C})$ such that $f(D-S) \not \subset H$ and $\left\{\nu\left(f^{(p)}, H\right)\right\}$ is a convergent sequence of divisors on $D$, then $\left\{f^{(p)}\right\}$ is meromorphically convergent on $D$.

Proof. Without loss of generality we may assume that $D=B(R)$, $0 \notin S$ and $\left\{f^{(p)}\right\}$ meromorphically converges on $B(R)-S$ to a meromorphic mapping $f: B(R)-S \rightarrow \mathbf{P}^{N}(\mathbf{C})$ with $f(0) \notin H$.

Let $x_{0}$ be any point of $S$. By [S, Theorem 2.7], for any $r(0<r<\tilde{R}=$ $\left.R-\left\|x_{0}\right\|\right)$, we can choose holomorphic functions $h^{(p)} \not \equiv 0$ and $h \not \equiv 0$ on $B\left(x_{0}, r\right)$ such that $\nu\left(f^{(p)}, H\right)=\nu_{h^{(p)}}, \nu=\nu_{h}$ for the limit $\nu$ of $\left\{\nu\left(f^{(p)}, H\right)\right\}$ and $\left\{h^{(p)}\right\}$ converges uniformly on compact subsets of $B\left(x_{0}, r\right)$ to $h$. Then, each $f^{(p)}$ has an admissible representation on $B\left(x_{0}, r\right)$

$$
f^{(p)}=\left(f_{0}^{(p)}: f_{1}^{(p)}: \cdots: f_{N}^{(p)}\right)
$$

with suitable holomorphic functions $f_{i}^{(p)}(0 \leq i \leq N)$ on $B\left(x_{0}, r\right)$.

Let $x$ be a point in $B\left(x_{0}, r\right)-(S \cup\{h=0\})$. Choose a simply connected relatively compact neighborhood $W_{x}$ of $x$ in $B\left(x_{0}, r\right)-(S \cup\{h=0\})$ such that there exists a sequence $\left\{u_{x}^{(p)}\right\}$ of nonvanishing holomorphic functions on $W_{x}$ such that $\left\{u_{x}^{(p)} f_{i}^{(p)}\right\} \rightarrow f_{i}^{x}(0 \leq i \leq N)$ on $W_{x}$ and $f=\left(f_{0}^{x}: f_{1}^{x}: \cdots\right.$ : 
$\left.f_{N}^{x}\right)$ on $W_{x}$. It may be assumed that $h^{(p)}(p \geq 1)$ has no zero on $W_{x}$. Let $Q$ be the defining polynomial of $H$. We have $Q\left(f^{(p)}\right)=Q\left(f_{0}^{(p)}, \ldots, f_{N}^{(p)}\right)=$ $v^{(p)} h^{(p)}$, where $v^{(p)}$ is a nonvanishing holomorphic function on $B\left(x_{0}, r\right)$. This implies that $Q\left(u_{x}^{(p)} f_{0}^{(p)}, \ldots, u_{x}^{(p)} f_{N}^{(p)}\right) \neq 0$ on $W_{x}$. Since $Q$ is a homogeneous polynomial, $Q\left(u_{x}^{(p)} f_{0}^{(p)}, \ldots, u_{x}^{(p)} f_{N}^{(p)}\right) \rightarrow Q\left(f_{0}^{x}, \ldots, f_{N}^{x}\right)$ on $W_{x}$. Since $f(B(R)-S) \not \subset H$, it implies that $Q\left(f_{0}^{x}, \ldots, f_{N}^{x}\right) \not \equiv 0$ on $W_{x}$, and hence $Q\left(f_{0}^{x}, \ldots, f_{N}^{x}\right) \neq 0$ on $W_{x}$. Assume that $Q$ has degree $d$. Since

$$
\begin{gathered}
Q\left(u_{x}^{(p)} f_{0}^{(p)}, \ldots, u_{x}^{(p)} f_{N}^{(p)}\right) \text { tends to } Q\left(f_{0}^{x}, \ldots, f_{N}^{x}\right) \text { on } W_{x} \text { and } \\
Q\left(u_{x}^{(p)} f_{0}^{(p)}, \ldots, u_{x}^{(p)} f_{N}^{(p)}\right)=\left(u_{x}^{(p)}\right)^{d} \cdot v^{(p)} \cdot h^{(p)}
\end{gathered}
$$

it follows that $\left(u_{x}^{(p)}\right)^{d} \cdot v^{(p)} \cdot h^{(p)}$ tends to $Q\left(f_{0}^{x}, \ldots, f_{N}^{x}\right)$ on $W_{x}$. Since $v^{(p)} \neq$ 0 on $B\left(x_{0}, r\right), v^{(p)}=\left(k^{(p)}\right)^{d}$, where $k^{(p)}$ is a nonvanishing holomorphic mapping on $B\left(x_{0}, r\right)$. We have

$$
\left(u_{x}^{(p)}\right)^{d} \cdot\left(k^{(p)}\right)^{d}=\left(u_{x}^{(p)} \cdot k^{(p)}\right)^{d} \rightarrow \frac{Q\left(f_{0}^{x}, \ldots, f_{N}^{x}\right)}{h} \text { on } W_{x} .
$$

Define

$$
F^{d}:=\frac{Q\left(f_{0}^{x}, \ldots, f_{N}^{x}\right)}{h} \text { on } W_{x} .
$$

Obviously $F^{d} \neq 0$ on $W_{x}$. So $\left(u_{x}^{(p)} \cdot k^{(p)}\right)^{d} \rightarrow F^{d}$ on $W_{x}$, hence $\left(u_{x}^{(p)} \cdot k^{(p)} / F\right)^{d}$ tends to 1 on $W_{x}$. Therefore, there exist (or empty) infinite subsets $\left\{N_{j}^{x}\right\}_{j=0}^{d-1}$ of $\mathbf{N}$ such that

$$
\begin{gathered}
\mathbf{N} \text { is a disjoint union of sets } N_{j}^{x} \text { and } \\
\left\{\frac{u_{x}^{(p)} \cdot k^{(p)}}{F}\right\}_{p \in N_{j}^{x}} \rightarrow \theta_{j}=e^{i \cdot 2 \pi j / d} \text { for each } 0 \leq j \leq d-1 .
\end{gathered}
$$

This implies that $\left\{f_{i}^{(p)} / k^{(p)}\right\}_{p \in N_{j}^{x}} \rightarrow F_{i}^{x} / \theta_{j}$ on $W_{x}$, where $F_{i}^{x}=f_{i}^{x} / F$ on $W_{x}$.

Take $a \in B\left(x_{0}, r\right)-(S \cup\{h=0\})$. Then $\left\{f_{i}^{(p)} / k^{(p)}\right\}_{p \in N_{j}^{a}} \rightarrow F_{i}^{a} / \theta_{j}$ on $W_{a}$ for each $0 \leq j \leq d-1$.

Take $b \in B\left(x_{0}, r\right)-(S \cup\{h=0\})$ such that $W_{a} \cap W_{b} \neq \emptyset$. We will prove that $\left\{f_{i}^{(p)} / k^{(p)}\right\}_{p \in N_{j}^{a}} \rightarrow\left(F_{i}^{b} / \theta_{j}\right) \cdot c$ for each $0 \leq j \leq d-1$. Indeed, without loss of generality we may assume that $f_{0}^{a} \not \equiv 0$ on $W_{a}$. Then $f_{0}^{x} \not \equiv 0$ on $W_{x}$ for each $x \in B\left(x_{0}, r\right)-(S \cup\{h=0\})$. Hence $F_{0}^{x} \not \equiv 0$ on $W_{x}$ for each $x \in B\left(x_{0}, r\right)-(S \cup\{h=0\})$. 
Consider $\left|N_{j}^{a}\right|=\infty$, where $|\cdot|$ denotes the cardinality of a set.

Assume that there exist $N_{1}^{b}, N_{2}^{b}$ such that $\left|\tilde{N}=N_{j}^{a} \cap N_{1}^{b}\right|=\mid \tilde{\tilde{N}}=N_{j}^{a} \cap$ $N_{2}^{b} \mid=\infty$. Since $\left\{f_{0}^{(p)} / k^{(p)}\right\}_{p \in \tilde{N} \subset N_{1}^{b}} \rightarrow F_{0}^{b} / \theta_{1}$ on $W_{b}$ and $\left\{f_{0}^{(p)} / k^{(p)}\right\}_{p \in \tilde{N} \subset N_{j}^{a}}$ $\rightarrow F_{0}^{a} / \theta_{j}$ on $W_{a}$, we have $F_{0}^{b} / \theta_{1}=F_{0}^{a} / \theta_{j}$ on $W_{a} \cap W_{b}$. Similarly, $F_{0}^{b} / \theta_{2}=$ $F_{0}^{a} / \theta_{j}$ on $W_{a} \cap W_{b}$. This is a contradiction. Thus every infinite subset $N_{j}^{a}$ intersects and only intersects infinitely with the subset $N_{\alpha(j)}^{b}$. Moreover, $\left|N_{j}^{a} \Delta N_{\alpha(j)}^{b}\right|<\infty$.

From this it follows that there exists a bijection $\alpha:\{0,1, \ldots, d-1\} \rightarrow$ $\{0,1, \ldots, d-1\}$ such that

$$
\begin{gathered}
N_{j}^{a}=\emptyset \text { if and only if } N_{\alpha(j)}^{b}=\emptyset, \\
\text { if }\left|N_{j}^{a}\right|=\infty \text { then }\left|N_{j}^{a} \Delta N_{\alpha(j)}^{b}\right|<\infty .
\end{gathered}
$$

On the other hand, since $\left\{f_{0}^{(p)} / k^{(p)}\right\}_{p \in N_{j}^{a} \cap N_{\alpha(j)}^{b}} \rightarrow F_{0}^{a} / \theta_{j}$ on $W_{a}$ and $\left\{f_{0}^{(p)} / k^{(p)}\right\}_{p \in N_{j}^{a} \cap N_{\alpha(j)}^{b}} \rightarrow F_{0}^{b} / \theta_{\alpha(j)}$ on $W_{b}$, we have $F_{0}^{a} / \theta_{j}=F_{0}^{b} / \theta_{\alpha(j)}$ on $W_{a} \cap W_{b}$. This means that $F_{0}^{a}=F_{0}^{b} \circ\left(\theta_{j} / \theta_{\alpha(j)}\right)$ on $W_{a} \cap W_{b}$ for each $0 \leq j \leq d-1$, and hence, $\theta_{j} / \theta_{\alpha(j)} \equiv c_{b}$ : constant for each $0 \leq j \leq$ $d-1$. It implies that $\left\{f_{i}^{(p)} / k^{(p)}\right\}_{p \in N_{j}^{a} \cap N_{\alpha(j)}^{b}} \rightarrow F_{i}^{b} / \theta_{\alpha(j)}=\left(F_{i}^{b} / \theta_{j}\right) \cdot c_{b}$ on $W_{b}$, and hence, $\left\{f_{i}^{(p)} / k^{(p)}\right\}_{p \in N_{j}^{a}} \rightarrow\left(F_{i}^{b} / \theta_{j}\right) \cdot c_{b}$ on $W_{b}$. Using the finite cover argument, we have $\left\{f_{i}^{(p)} / k^{(p)}\right\}_{p \in N_{j}^{a}} \rightarrow\left(F_{i}^{x} / \theta_{j}\right) \cdot c_{x}$ on $W_{x}$ for each $x \in B\left(x_{0}, r\right)-(S \cup\{h=0\})$ and for each $0 \leq j \leq d-1$. For $p \in N_{j}^{a}$ put $\tilde{f}_{i}^{(p)}=f_{i}^{(p)} \cdot\left(\theta_{j} / k^{(p)}\right)(0 \leq i \leq N)$. Then $f^{(p)}=\left(\tilde{f}_{0}^{(p)}, \ldots, \tilde{f}_{N}^{(p)}\right)$ for all $p \in N_{j}^{a}$ and $0 \leq j \leq d-1$ and $\left\{\tilde{f}_{i}^{(p)}\right\}_{p=1}^{\infty} \rightarrow F_{i}^{x} \cdot c_{x}$ on $W_{x}$ for each $0 \leq i \leq N$. Note that if $W_{x} \cap W_{y} \neq \emptyset\left(x, y \in B\left(x_{0}, r\right)-(S \cup\{h=0\})\right)$ then $F_{i}^{x} \cdot c_{x}=F_{i}^{y} \cdot c_{y}$ for each $0 \leq i \leq N$.

Define the function $F_{i}: B\left(x_{0}, r\right)-(S \cup\{h=0\}) \rightarrow \mathbf{C}$ given by $\left.F_{i}\right|_{W_{x}}=$ $F_{i}^{x} \cdot c_{x}$. Then $\left\{\tilde{f}_{i}^{(p)}\right\}_{p=1}^{\infty} \rightarrow F_{i}$ on $B\left(x_{0}, r\right)-(S \cup\{h=0\})$ for each $0 \leq i \leq N$. We now prove that the sequence $\left\{f^{(p)}\right\}_{p=1}^{\infty}$ meromorphically converges on $B\left(x_{0}, r\right)$ to some meromorphic mapping $\tilde{F}=\left(\tilde{F}_{0}, \ldots, \tilde{F}_{N}\right)$. Indeed, let $z_{0}$ be any point of $S_{1}=S \cup\{h=0\}$. Since $\Lambda^{2 n-1}\left(S_{1}\right)=0$, there exists a complex line $l_{z_{0}}$ passing through $z_{0}$ such that $\Lambda^{1}\left(S_{1} \cap l_{z_{0}}\right)=0$. Put $l_{z_{0}}=\left\{z_{0}+z \cdot u: z \in \mathbf{C}\right\}$. Then there exists $R>0$ such that

$$
\mathcal{C}_{0}=\left\{z_{0}+R \cdot e^{i \theta} \cdot u: \theta \in[0,2 \pi]\right\}
$$


satisfying $\mathcal{C}_{0} \subset B\left(x_{0}, r\right)$ and $\mathcal{C}_{0} \cap S_{1}=\emptyset$. By the maximum principle, it implies that the sequence $\left\{\tilde{f}_{i}^{(p)}\left(z_{0}\right)\right\}$ converges. Put $\lim _{p \rightarrow \infty} \tilde{f}_{i}^{(p)}\left(z_{0}\right)=$ $\tilde{F}_{i}\left(z_{0}\right)$. This means that the mapping $F_{i}$ extendes over $B\left(x_{0}, r\right)$ to the mapping $\tilde{F}_{i}$.

We now prove that the sequence $\left\{\tilde{f}_{i}^{(p)}(z)\right\}_{p=1}^{\infty}$ converges uniformly on compact subsets of $B\left(x_{0}, r\right)$ to $\tilde{F}_{i}(z)$. Indeed, assume that $\left\{z_{j}\right\} \subset B\left(x_{0}, r\right)$ converges to $z_{0} \in B\left(x_{0}, r\right)$. As above, there exists a circle $\mathcal{C}_{0}=\left\{z_{0}+R \cdot e^{i \theta} \cdot u\right.$ : $\theta \in[0,2 \pi]\} \subset B\left(x_{0}, r\right)$ such that $\mathcal{C}_{0} \cap S_{1}=\emptyset$. Since $\mathcal{C}_{0}$ is a compact subset of $B\left(x_{0}, r\right)-S_{1}$, there exists $\epsilon_{0}>0$ such that

$$
V\left(\mathcal{C}_{0}, \epsilon_{0}\right)=\left\{z \in \mathbf{C}^{n}: \operatorname{dist}\left(z, \mathcal{C}_{0}\right)<\epsilon_{0}\right\} \Subset B\left(x_{0}, r\right)-S_{1} .
$$

Consider the circles $\mathcal{C}_{j}=\left\{z_{j}+R \cdot e^{i \theta} \cdot u: \theta \in[0,2 \pi]\right\}$. It is easy to see that $\operatorname{dist}\left(\mathcal{C}_{0}, \mathcal{C}_{j}\right)=\left\|z_{j}-z_{0}\right\| \rightarrow 0$ as $j \rightarrow \infty$. Thus, without loss of generality, we may assume that $\mathcal{C}_{j} \subset V\left(\mathcal{C}_{0}, \epsilon_{0}\right) \Subset B\left(x_{0}, r\right)-S_{1}$. By the hypothesis, $\forall \epsilon>0, \exists N=N(\epsilon)$ such that

$$
\sup \left\{\left\|\tilde{f}_{i}^{(p)}(z)-F_{i}(z)\right\|: z \in V\left(\mathcal{C}_{0}, \epsilon_{0}\right), p \geq N\right\}<\epsilon
$$

By the maximum principle, we have $\limsup _{j \rightarrow \infty}\left\|\tilde{f}_{i}^{(j)}\left(z_{j}\right)-F_{i}\left(z_{j}\right)\right\|=0$. This implies that the sequence $\left\{\tilde{f}_{i}^{(p)}\right\}_{p=1}^{\infty}$ converges uniformly on compact subsets of $B\left(x_{0}, r\right)$ to $\tilde{F}_{i}$.

LEMMA 3.5. Let $\left\{f^{(p)}\right\}$ be a sequence of meromorphic mappings of a domain $D$ in $\mathbf{C}^{n}$ into $\mathbf{P}^{N}(\mathbf{C})$ and let $S$ be a closed subset of $D$ with $\Lambda^{2 n-1}(S)=0$. Suppose that $\left\{f^{(p)}\right\}$ meromorphically converges on $D-S$ to a meromorphic mapping $f$ of $D-S$ into $\mathbf{P}^{N}(\mathbf{C})$. Suppose that, for each $f^{(p)}$, there exist $N+1$ hypersurfaces $H_{1}\left(f^{(p)}\right), \ldots, H_{N+1}\left(f^{(p)}\right)$ in $\mathbf{P}^{N}(\mathbf{C})$, where the hypersurfaces $H_{i}\left(f^{(p)}\right)$ may depend on $f^{(p)}$, such that the following two conditions are satisfied.

$i) \inf \left\{D\left(H_{1}\left(f^{(p)}\right), \ldots, H_{N+1}\left(f^{(p)}\right)\right): p \geq 1\right\}>0$.

ii) The $2(n-1)$-dimensional Lebesgue areas of $f^{(p)^{-1}}\left(H_{k}\left(f^{(p)}\right)\right) \cap E$ $(1 \leq k \leq N+1 ; p \geq 1)$ with counting multiplicities are bounded above for any fixed compact subset $E$ of $D$.

Then $\left\{f^{(p)}\right\}$ has a meromorphically convergent subsequence on D.

Proof. We also assume that $D=B(R), 0 \notin S$ and $f(0) \notin H$. By the hypothesis ii) in Lemma 3.5 and by Lemma 3.3 , we can assume that $\nu\left(f^{(p)}, H_{k}\left(f^{(p)}\right)\right)$ converges in $\mathcal{D}^{+}(B(R))(1 \leq k \leq N+1)$. 
As in the proof of Lemma 3.4, there exist holomorphic mappings $h_{k}^{(p)} \not \equiv$ 0 and $h_{k} \not \equiv 0$ on $B(r)$ such that $\nu\left(f^{(p)}, H_{k}\left(f^{(p)}\right)\right)=\nu_{h_{k}^{(p)}}, \nu_{k}=\nu_{h_{k}}$ for the limit $\nu_{k}$ of $\left\{\nu\left(f^{(p)}, H_{k}\left(f^{(p)}\right)\right)\right\}$ for each $1 \leq k \leq N+1(0<r<R)$ and $\left\{h_{k}^{(p)}\right\}$ converges uniformly on compact subsets of $B(r)$ to $h_{k}(1 \leq k \leq N+1)$. Take $x \in B(r)-\left(S \cup \bigcup_{k=1}^{N+1}\left\{h_{k}=0\right\}\right)$. Choose a simply connected relatively compact neighborhood $W_{x}$ of $x$ in $B(r)-\left(S \cup \bigcup_{k=1}^{N+1}\left\{h_{k}=0\right\}\right)$. Then, each $f^{(p)}$ has an admissible representation $f^{(p)}=\left(f_{0}^{(p)}: f_{1}^{(p)}: \cdots: f_{N}^{(p)}\right)$ with suitable holomorphic functions $f_{i}^{(p)}(0 \leq i \leq N)$ on $B(r)$. At the same time, there exist representations of $f^{(p)}(p \geq 1)$ on $W_{x}: f^{(p)}=\left(u^{(p)} f_{0}^{(p)}: u^{(p)} f_{1}^{(p)}\right.$ : $\left.\cdots: u^{(p)} f_{N}^{(p)}\right)$ such that $u^{(p)}$ are nonvanishing holomorphic functions on $W_{x}$ and $u^{(p)} f_{i}^{(p)} \rightarrow f_{i}(0 \leq i \leq N)$ on $W_{x}$ and $\left(f_{0}: f_{1}: \cdots: f_{N}\right)$ is a representation of $f$ on $W_{x}$.

For each $1 \leq k \leq N+1$, let $Q_{k}^{d_{k}}\left(f^{(p)}\right)$ be the defining polynomial of $H_{k}\left(f^{(p)}\right)$, where the superscript $d_{k}$ indicates the degree of the polynomial: $\operatorname{deg} Q_{k}^{d_{k}}\left(f^{(p)}\right)=d_{k}$. Put $Q_{k}^{(p)}:=Q_{k}^{d_{k}}\left(f^{(p)}\right)$. Then there exists a subsequence $\left\{Q_{k}^{\left(p_{j}\right)}\right\}_{j=1}^{\infty}$ of $\left\{Q_{k}^{(p)}\right\}_{p=1}^{\infty}$ such that $\left\{Q_{k}^{\left(p_{j}\right)}\right\}_{j=1}^{\infty}$ converges uniformly on compact subsets of $\mathbf{C}^{N+1}$ to a homogeneous polynomial $Q_{k}$. Without loss of generality we can assume $\left\{Q_{k}^{(p)}\right\}_{p=1}^{\infty}$ converges uniformly on compact subsets of $\mathbf{C}^{N+1}$ to $Q_{k}$ as $p \rightarrow \infty$. Therefore $Q_{k}^{(p)}\left(u^{(p)} f_{0}^{(p)}, \ldots, u^{(p)} f_{N}^{(p)}\right) \rightarrow$ $Q_{k}\left(f_{0}, \ldots, f_{N}\right)$ on compact subsets of $W_{x}$ as $p \rightarrow \infty$. Let

$$
\begin{aligned}
H_{k}:=\left\{\left(z_{0}: z_{1}: \cdots: z_{N}\right) \in \mathbf{P}^{N}(\mathbf{C}): Q_{k}\left(z_{0}, z_{1}, \ldots, z_{N}\right)=0\right\} & \\
& (1 \leq k \leq N+1) .
\end{aligned}
$$

Then $D\left(H_{1}, \ldots, H_{N+1}\right) \geq \liminf _{p \rightarrow \infty} D\left(H_{1}\left(f^{(p)}\right), \ldots, H_{N+1}\left(f^{(p)}\right)\right)$. By the hypothesis, we have $\inf \left\{D\left(H_{1}\left(f^{(p)}\right), \ldots, H_{N+1}\left(f^{(p)}\right)\right): p \geq 1\right\}>0$. Thus $D\left(H_{1}, \ldots, H_{N+1}\right)>0$, i.e. hypersurfaces $H_{1}, H_{2}, \ldots, H_{N+1}$ have no common point. Hence, there exists $k_{0} \in\{1, \ldots, N+1\}$ such that $f(D-$ $S) \not \subset H_{k_{0}}$. Since $Q_{k_{0}}^{(p)}\left(u^{(p)} f_{0}^{(p)}, \ldots, u^{(p)} f_{N}^{(p)}\right)$ converges uniformly on compact subsets of $W_{x}$ to $Q_{k_{0}}\left(f_{0}, \ldots, f_{N}\right)$ and by the same argument as in the proof of Lemma 3.4, it implies that $\left\{f^{(p)}\right\}$ meromorphically converges on $D$.

\subsection{Proof of Theorem A}

Without loss of generality, we may assume that $D=\Delta^{n}$. Let $\left\{f_{i}\right\} \subset \mathcal{F}$. By Lemmas 3.2, 3.3 and by passing if necessary to a subsequence, we may assume that the sequence $\left\{f_{i}\right\}$ satisfies

$$
\lim _{i \rightarrow \infty} f_{i}^{-1}\left(H_{k}\left(f_{i}\right)\right)=S_{k} \quad(1 \leq k \leq N+1)
$$


as a sequence of closed subsets of $\Delta^{n}$, where $S_{k}$ are either empty or pure $(n-1)$-dimensional analytic sets in $\Delta^{n}$, and

$$
\lim _{i \rightarrow \infty} f_{i}^{-1}\left(H_{k}\left(f_{i}\right)\right)-S=S_{k} \quad(N+2 \leq k \leq q),
$$

as a sequence of closed subsets of $\Delta^{n}-S$, where $S_{k}$ are either empty or a pure $(n-1)$-dimensional analytic sets in $\Delta^{n}-S$. Let $E:=\bigcup_{k=1}^{q} S_{k}-S$. Then $E$ is either empty or a pure $(n-1)$-dimensional analytic set in $\Delta^{n}-S$. For each $1 \leq k \leq q$, let $Q_{k}^{d_{k}}\left(f_{i}\right)$ be the defining polynomial of $H_{k}\left(f_{i}\right)$. Put $Q_{k}^{(i)}:=Q_{k}^{d_{k}}\left(f_{i}\right)$. Without loss of generality we can assume $\left\{Q_{k}^{(i)}\right\}_{i=1}^{\infty}$ converges uniformly on compact subsets of $\mathbf{C}^{N+1}$ to $Q_{k}$ as $i \rightarrow \infty$. Let

$$
H_{k}:=\left\{\left(z_{0}: z_{1}: \cdots: z_{N}\right) \in \mathbf{P}^{N}(\mathbf{C}): Q_{k}\left(z_{0}, z_{1}, \ldots, z_{N}\right)=0\right\} \quad(1 \leq k \leq q) .
$$

For any fixed point $z_{0}$ in $\left(\Delta^{n}-S\right)-E$, choose a relatively compact neighborhood $U_{z_{0}}$ in $\left(\Delta^{n}-S\right)-E$. Then $\left\{\left.f_{i}\right|_{U_{z_{0}}}\right\} \subset \operatorname{Hol}\left(U_{z_{0}}, \mathbf{P}^{N}(\mathbf{C})\right)$. We now prove that the family $\left\{\left.f_{i}\right|_{U_{z_{0}}}\right\}$ is a holomorphically normal family. Indeed, suppose that the family $\left\{\left.f_{i}\right|_{U_{z_{0}}}\right\}$ is not holomorphically normal. By [TTH, Theorem 2.5], there exist $p_{0} \in U_{z_{0}},\left\{p_{j}\right\} \subset U_{z_{0}}$ with $p_{j} \rightarrow p_{0}$, $\left\{\rho_{j}\right\} \subset(0, \infty)$ with $\rho_{j} \rightarrow 0^{+}$such that the sequence of holomorphic maps

$$
g_{j}(z)=f_{j}\left(p_{j}+\rho_{j} z\right): \Delta_{r_{j}}^{n} \rightarrow \mathbf{P}^{N}(\mathbf{C}) \quad\left(r_{j} \uparrow \infty\right)
$$

converges uniformly on compact subsets of $\mathbf{C}^{n}$ to a nonconstant holomorphic map $g: \mathbf{C}^{n} \rightarrow \mathbf{P}^{N}(\mathbf{C})$. Since $\left\{g_{j}\right\}$ converges uniformly on compact subsets of $\mathbf{C}^{n}$ to $g$, it follows that there exist admissible representations $\tilde{g}_{j}=\left(g_{j}^{0}, \ldots, g_{j}^{N}\right)$ and $\tilde{g}=\left(g^{0}, \ldots, g^{N}\right)$ of $g_{j}$ and $g$, respectively, such that $\left\{\tilde{g}_{j}\right\}$ converges uniformly on compact subsets of $\mathbf{C}^{n}$ to $\tilde{g}$. This implies that $\left\{Q_{k}^{(j)} \circ \tilde{g}_{j}\right\}$ converges uniformly on compact subsets of $\mathbf{C}^{n}$ to $Q_{k} \circ \tilde{g}$. Thus, by the Hurwitz's theorem, one of the following two assertions holds:

i) $Q_{k} \circ \tilde{g} \neq 0$ on $\mathbf{C}^{n}$, i.e. $g\left(\mathbf{C}^{n}\right) \cap H_{k}=\emptyset$.

ii) $Q_{k} \circ \tilde{g} \equiv 0$ on $\mathbf{C}^{n}$, i.e. $g\left(\mathbf{C}^{n}\right) \subset H_{k}$.

Hence there exists a subset $I \subset\{1,2, \ldots, q\}$ such that $g\left(\mathbf{C}^{n}\right) \subset$ $\left(\bigcap_{i \in I} H_{i}\right) \backslash\left(\bigcup_{i \notin I} H_{i}\right)$.

By [No-Wi, Corollary $1.4(\mathrm{ii})],\left(\bigcap_{i \in I} H_{i}\right) \backslash\left(\bigcup_{i \notin I} H_{i}\right)$ is hyperbolic, and hence $g$ is constant. This is a contradiction. Thus $\left\{f_{i}\right\}$ is a holomorphically normal family on $U_{z_{0}}$. Therefore, by the usual diagonal argument, we can find a subsequence (again denoted by $\left\{f_{i}\right\}$ ) which converges uniformly on compact subsets of $\left(\Delta^{n}-S\right)-E$ to a holomorphic mapping $f$ 
of $\left(\Delta^{n}-S\right)-E$ into $\mathbf{P}^{N}(\mathbf{C})$. By Lemma $3.5,\left\{f_{i}\right\}$ has a meromorphically convergent subsequence (again denoted by $\left\{f_{i}\right\}$ ) on $\Delta^{n}-S$ and again by Lemma 3.5, $\left\{f_{i}\right\}$ has a meromorphically convergent subsequence on $\Delta^{n}$. Then $\mathcal{F}$ is a meromorphically normal family on $\Delta^{n}$. The proof of Theorem A is completed.

Remark 3.7. By the same argument as the proof of Theorem A, we get an another criterion for meromorphic normality.

TheOREM $\mathrm{A}^{\prime}$. Let $\mathcal{F}$ be a family of meromorphic mappings of a domain $D$ in $\mathbf{C}^{n}$ into $\mathbf{P}^{N}(\mathbf{C})$. Suppose that there exist $q \geq 2 N+1$ hypersurfaces $H_{1}, \ldots, H_{q}$ in $\mathbf{P}^{N}(\mathbf{C})$ with

$$
D\left(H_{1}, \ldots, H_{q}\right)>0 \text { and } f(D) \not \subset H_{i} \quad(1 \leq i \leq N+1),
$$

such that the following three conditions are satisfied.

i) For any fixed compact subset $K$ of $D$, the $2(n-1)$-dimensional Lebesgue areas of $f^{-1}\left(H_{i}\right) \cap K(1 \leq i \leq N+1)$ with counting multiplicities for all $f$ in $\mathcal{F}$ are bounded above.

ii) There exists a closed subset $S$ of $D$ with $\Lambda^{2 n-1}(S)=0$ such that for any fixed compact subset $K$ of $D-S$, the $2(n-1)$-dimensional Lebesgue areas of

$$
\left\{z \in \operatorname{supp} \nu\left(f, H_{j}\right) ; \nu\left(f, H_{j}\right)(z)<m_{j}\right\} \cap K \quad(N+2 \leq j \leq q)
$$

regardless of multiplicities for all $f$ in $\mathcal{F}$, are bounded above, where $\left\{m_{j}\right\}_{j=N+2}^{q}$ are fixed positive integers and may be $\infty$.

iii) Any holomorphic mapping $\varphi: \mathbf{C} \rightarrow \mathbf{P}^{N}(\mathbf{C})$ which intersects $H_{j}$ with mutiplicitiy at least $m_{j}(N+2 \leq j \leq q)$, must be constant.

Then $\mathcal{F}$ is a meromorphically normal family on $D$.

We now give a corollary of Theorem A.

Corollary 3.8. Let $\mathcal{F}$ be a family of holomorphic mappings of a domain $D$ in $\mathbf{C}^{n}$ into $\mathbf{P}^{N}(\mathbf{C})$. Suppose that for each $f \in \mathcal{F}$, there exist $q \geq 2 N+1$ hypersurfaces $H_{1}(f), H_{2}(f), \ldots, H_{q}(f)$ in $\mathbf{P}^{N}(\mathbf{C})$ with

$$
\inf \left\{D\left(H_{1}(f), \ldots, H_{q}(f)\right): f \in \mathcal{F}\right\}>0,
$$

where $q$ is independent of $f$, but the hypersurfaces $H_{i}(f)$ may depend on $f$, such that the following two conditions are satisfied 
i) $f(D) \cap H_{i}(f)=\emptyset(1 \leq i \leq N+1)$ for any $f$ in $\mathcal{F}$.

ii) There exists a closed subset $S$ of $D$ with $\Lambda^{2 n-1}(S)=0$ such that for any fixed compact subset $K$ of $D-S$, the $2(n-1)$-dimensional Lebesgue areas of $f^{-1}\left(H_{i}(f)\right) \cap K(N+2 \leq i \leq q)$ with counting multiplicities for all $f \in \mathcal{F}$ are bounded above.

Then $\mathcal{F}$ is a holomorphically normal family on $D$.

In order to prove this corollary, we need the following lemma.

LEMMA 3.9. Let $\left\{f^{(p)}\right\}$ be a meromorphically convergent sequence of holomorphic mappings of polydisc $\Delta^{n}$ in $\mathbf{C}^{n}$ into $\mathbf{P}^{N}(\mathbf{C})$. If for each $f^{(p)}$, there exist $N+1$ hypersurfaces $H_{1}\left(f^{(p)}\right), \ldots, H_{N+1}\left(f^{(p)}\right)$ in $\mathbf{P}^{N}(\mathbf{C})$ such that

$$
\inf \left\{D\left(H_{1}\left(f^{(p)}\right), \ldots, H_{N+1}\left(f^{(p)}\right)\right): p \geq 1\right\}>0
$$

and

$$
f^{(p)}\left(\Delta_{n}\right) \cap H_{k}\left(f^{(p)}\right)=\emptyset \quad(1 \leq k \leq N+1 ; p \geq 1),
$$

where the hypersurfaces $H_{i}\left(f^{(p)}\right)$ may depend on $f^{(p)}$.

Then $\left\{f^{(p)}\right\}$ converges uniformly on compact subsets of $\Delta^{n}$ to a holomorphic mapping of $\Delta^{n}$ into $\mathbf{P}^{N}(\mathbf{C})$.

Proof. Let $z_{0} \in \Delta_{n}$. Then every $f^{(p)}$ has an admissible representation

$$
f^{(p)}(z)=\left(f_{0}^{(p)}(z): f_{1}^{(p)}(z): \cdots: f_{N}^{(p)}(z)\right) \quad(p \geq 1)
$$

on a fixed neighborhood $U\left(z_{0}\right)$ of $z_{0}$ such that $f^{(p)}$ converges uniformly on compact subsets of $U\left(z_{0}\right)$ to $f(z)=\left(f_{0}(z): f_{1}(z): \cdots: f_{N}(z)\right), f(z) \not \equiv 0$ on $U\left(z_{0}\right)$ (by Lemma 3.5).

We now show that $f(z) \neq 0$ everywhere on $U\left(z_{0}\right)$ and hence $\left\{f^{(p)}\right\}$ converges uniformly on compact subsets of $\Delta^{n}$ to a holomorphic mapping of $\Delta^{n}$ into $\mathbf{P}^{N}(\mathbf{C})$. As in the proof of Lemma 3.5, there exists $k_{0}$ such that $f\left(\Delta^{n}\right) \not \subset H_{k_{0}}$. By denoting as in the proof of Lemma 3.5, we have $Q_{k_{0}}^{(p)}\left(f_{0}^{(p)}, \ldots, f_{N}^{(p)}\right)$ converges uniformly on compact subsets of $U$ to $Q_{k_{0}}\left(f_{0}, \ldots, f_{N}\right)$ as $p$ tends to infinity. Since $f^{(p)}\left(\Delta^{n}\right) \cap H_{k}\left(f^{(p)}\right)=\emptyset$ $(1 \leq k \leq N+1 ; p \geq 1)$, and $Q_{k_{0}}^{(p)}\left(f_{0}^{(p)}(z), \ldots, f_{N}^{(p)}(z)\right) \neq 0$ for all $z \in \Delta^{n}$, it implies that $Q_{k_{0}}^{(p)}\left(f_{0}^{(p)}(z), \ldots, f_{N}^{(p)}(z)\right) \neq 0$ on $U$. On the other hand, since $Q_{k_{0}}\left(f_{0}, \ldots, f_{N}\right) \not \equiv 0$ on $U, Q_{k_{0}}\left(f_{0}, \ldots, f_{N}\right) \neq 0$ everywhere on $U$. This implies that, for any $z \in U$, there exists $l \in\{0, \ldots, N\}$ such that $f_{l}(z) \neq 0$. Thus $f$ is a holomorphic mapping on $U$ and hence, $\left\{f^{(p)}\right\}$ converges uniformly on compact subsets of $\Delta^{n}$ to a holomorphic mapping $f: \Delta^{n} \rightarrow \mathbf{P}^{N}(\mathbf{C})$. 


\subsection{Proof of Corollary 3.11}

By Theorem $\mathrm{A}, \mathcal{F}$ is a meromorphically normal family on $D$ and hence by Lemma $3.9, \mathcal{F}$ is a holomorphically normal family on $D$.

Lemma 3.11. Let $f$ be a meromorphic mapping from a domain $D$ in $\mathbf{C}^{n}$ into $\mathbf{P}^{N}(\mathbf{C})$. If there exist $q \geq 2 N+1$ hypersurfaces $H_{1}, \ldots, H_{q}$ in $\mathbf{P}^{N}(\mathbf{C})$ such that

$$
D\left(H_{1}, \ldots, H_{q}\right)>0 \text { and } f(D) \cap H_{j}=\emptyset \quad(1 \leq j \leq q) .
$$

Then $f$ is actually a holomorphic mapping from domain $D$ into $\mathbf{P}^{N}(\mathbf{C})$.

Proof. By [E-S] or $[\mathrm{Ru}], \mathbf{P}^{N}(\mathbf{C})-\bigcup_{i=1}^{2 n+1} H_{i}$ is hyperbolic. Since $f$ is a meromorphic mapping from $D$ into the hyperbolic space $\mathbf{P}^{N}(\mathbf{C})-\bigcup_{i=1}^{2 n+1} H_{i}$, by the theorem of Kodama (see [Ko, Theorem 6.3.19, p. 288]), $f$ is actually a holomorphic mapping.

\subsection{Proof of Theorem B}

Take any sequence $\left\{f_{i}\right\} \subset \mathcal{F}$. By the assumption and Lemmas 3.2 and 3.3 , we can find a subsequence (again denoted by $\left\{f_{i}\right\}$ ) such that $\lim _{i \rightarrow \infty} f_{i}^{-1}\left(H_{k}\left(f_{i}\right)\right)=S_{k}(1 \leq k \leq q)$ as a sequence of closed subsets of $D$, where $S_{k}$ are either empty or a pure $(n-1)$-dimensional analytic sets in $D$. Let $E:=\bigcup_{k=1}^{q} S_{k}$. Then $E$ is either empty or a pure $(n-1)$ dimensional analytic set of $D$, and hence $E$ is a nowhere dense analytic set of $D$. We now prove that $\left\{f_{i}\right\}_{i=1}^{\infty}$ has a compactly convergent subsequence on $D-E$.

For any fixed point $z_{0}$ in $D-E$, there exist an integer $i_{0}$ and a neighborhood $U_{z_{0}}$ in $D-E$ such that $f_{i}^{-1}\left(H_{k}\left(f_{i}\right)\right) \cap U_{z_{0}}=\emptyset(1 \leq k \leq q)$ for all $i \geq i_{0}$. By using Lemma 3.11, $\left\{f_{i}\right\}_{i=i_{0}}^{\infty}$ is a sequence of holomorphic mappings of $U_{z_{0}}$ into $\mathbf{P}^{N}(\mathbf{C})$ and by using again the argument in the proof of Theorem A, $\left\{f_{i}\right\}_{i=i_{0}}^{\infty}$ has a subsequence which converges uniformly on compact subsets of $U_{z_{0}}$ to a holomorphic mapping of $U_{z_{0}}$ into $\mathbf{P}^{N}(\mathbf{C})$. Therefore, by the usual diagonal argument, we can find a subsequence $\left\{f_{i_{j}}(z)\right\}$ so as to converge uniformly on compact subsets of $D-E$ to a holomorphic mapping of $D-E$ into $\mathbf{P}^{N}(\mathbf{C})$ and hence $\left\{f_{i_{j}}(z)\right\}$ is a quasi-regular on $D$. The proof of Theorem B is completed.

\section{§4. Extending holomorphic mappings into compact complex spa- ces}

Modifying the notions in [JK1], we give the following definition. 
Definition 4.1. Let $X$ be a complex space and $M$ a compact complex space. We say that the family $\mathcal{F} \subset \operatorname{Hol}(X, M)$ is uniformly normal if $\mathcal{F} \circ \operatorname{Hol}(\Delta, X):=\{f \circ \varphi: f \in \mathcal{F}$ and $\varphi \in \operatorname{Hol}(\Delta, X)\} \Subset \operatorname{Hol}(\Delta, M)$, and that a mapping $f \in \operatorname{Hol}(X, M)$ is a normal mapping if the family $\{f\}$ is uniformly normal. Here $\operatorname{Hol}(Y, Z)$ is the space of holomorphic mappings from a complex space $Y$ to a complex space $Z$ with the compact - open topology.

LEMMA 4.2. ([Noc]) Suppose that $q \geq 2 N+1$ hyperplanes $H_{1}, \ldots, H_{q}$ are given in general position in $\mathbf{P}^{N}(\mathbf{C})$, along with $q$ positive integers $m_{1}, \ldots, m_{q}\left(m_{i} \leq \infty, i=1, \ldots, q\right)$. If

$$
\sum_{i=1}^{q} \frac{1}{m_{i}}<\frac{q-(N+1)}{N}
$$

then there does not exist a nonconstant holomorphic mapping $f: \mathbf{C} \rightarrow$ $\mathbf{P}^{N}(\mathbf{C})$ such that $f$ intersects $H_{i}$ with multiplicity at least $m_{i}(i=1, \ldots, q)$.

Let $H$ be an analytic hypersurface of a compact complex space $M$. For every $a \in H$ denote by $\mathcal{F}_{a}$ the set of all pair $(U, \varphi)$, where $U$ is an open neighbourhood of $a$ in $M$ and $\varphi$ is a holomorphic function on $U$ such that $U \cap H=\{z \in U: \varphi(z)=0\}$. Recall that the holomorphic mapping $f$ on a domain $D \subset \mathbf{C}^{n}$ intersects the hypersurface $H$ with multiplicity at least $m(m<\infty)$ if $f(D) \not \subset H, f(D) \cap H \neq \emptyset$ and, for every $z_{0} \in D \cap f^{-1}(H)$ and for every $(U, \varphi) \in \mathcal{F}_{f\left(z_{0}\right)}, z_{0}$ is the zero with multiplicity $\geq m$ of the holomorphic function $\varphi(f(z))$. We say that the holomorphic mapping $f$ intersects the hypersurface $H$ with multiplicity $\infty$ on $D$ if $f(D) \subset H$ or $f(D) \cap H=\emptyset$.

Definition 4.3. Let $H_{1}, \ldots, H_{q}$ be $q$ hypersurfaces in a compact complex space $M$ and $m_{1}, \ldots, m_{q}$ be fixed positive integers $\left(m_{i} \leq \infty, i=\right.$ $1, \ldots, q)$. We say that the family $\left\{\left(H_{i}, m_{i}\right)\right\}_{i=1}^{q}$ has the D-property if every $f \in \operatorname{Hol}(\mathbf{C}, M)$ such that $f$ intersects $H_{i}$ with multiplicity at least $m_{i}$ $(i=1, \ldots, q)$ is constant.

It is easy to see that the family $\left\{\left(H_{i}, m_{i}\right)\right\}_{i=1}^{q}$ has the D-property iff any $f \in \operatorname{Hol}(\mathbf{C}, M)$ such that $\left.f\right|_{\mathbf{C}^{*}}$ intersects $H_{i}$ with multiplicity at least $m_{i}(i=1, \ldots, q)$, then $f$ is constant. 
ExAmples. - Suppose that $q \geq 2 N+1$ hyperplanes $H_{1}, \ldots, H_{q}$ are given in general position in $\mathbf{P}^{N}(\mathbf{C})$, along with $q$ positive integers $m_{1}, \ldots$, $m_{q}\left(m_{i} \leq \infty, i=1, \ldots, q\right)$ such that

$$
\sum_{i=1}^{q} \frac{1}{m_{i}}<\frac{q-(N+1)}{N} .
$$

Then, by Nochka's theorem (cf. Lemma 4.2), the family $\left\{\left(H_{i}, m_{i}\right)\right\}_{i=1}^{q}$ has the D-property.

- Let $\mathbf{H}_{\mathbf{0}} \rightarrow \mathbf{P}^{N}(\mathbf{C})$ be the hyperplane bundle and $H_{j} \in\left|\mathbf{H}_{\mathbf{0}}^{\mathbf{d}}\right|(1 \leq j \leq$ q) hyperbolic non-singular analytic hypersurfaces such that $H=\sum_{j=1}^{q} H_{j}$ has only normal crossings and $c_{1}([H])+c_{1}\left(K\left(\mathbf{P}^{N}(\mathbf{C})\right)\right)>0$. Let $m_{1}, \ldots, m_{q}$ $\left(m_{i} \leq \infty, i=1, \ldots, q\right)$ be positive integers such that

$$
\sum_{j=1}^{q}\left(1-\frac{1}{m_{j}}\right)>\frac{N+1}{d} .
$$

Then, by the Ramification Theorem [NO, Example 5.5.56, p. 217], the family $\left\{\left(H_{i}, m_{i}\right)\right\}_{i=1}^{q}$ has the D-property.

\subsection{Proof of Theorem $\mathrm{C}$}

Since the problem is local, we may assume that $\Omega=\Delta^{n}$ and $\Omega-S=$ $\left(\Delta^{*}\right)^{n}$. We first show that the family $\mathcal{F}:=\left\{f \circ \varphi: \varphi \in \operatorname{Hol}\left(\Delta,\left(\Delta^{*}\right)^{n}\right)\right\}$ is a holomorphically normal family.

Indeed, suppose that $\mathcal{F}$ is not normal. By using Zalcman's theorem $[\mathrm{AK}],[\mathrm{TTH}]$, there exist sequences $\left\{p_{j}\right\} \subset \Delta$ with $\left\{p_{j}\right\} \rightarrow p_{0} \in \Delta,\left\{f_{j}\right\} \subset$ $\mathcal{F},\left\{\rho_{j}\right\} \subset \mathbf{R}$ with $\rho_{j}>0$ and $\left\{\rho_{j}\right\} \rightarrow 0$ such that

$$
g_{j}(\xi)=f_{j}\left(p_{j}+\rho_{j} \xi\right), \quad \xi \in \mathbf{C}
$$

converges uniformly on compact subsets of $\mathbf{C}$ to a nonconstant holomorphic $\operatorname{map} g: \mathbf{C} \rightarrow M$.

Since $f$ intersects $H_{i}$ with multiplicity at least $m_{i}(i=1, \ldots, q)$, it is easy to see that $g_{j}$ also intersects $H_{i}$ with multiplicity at least $m_{i}(i=$ $1, \ldots, q ; j=1,2, \ldots)$.

By using Hurwitz theorem, it follows that $g$ intersects $H_{i}$ with multiplicity at least $m_{i}(i=1, \ldots, q)$. Since $\left\{\left(H_{i}, m_{i}\right)\right\}_{i=1}^{q}$ has the $D$-property, $g$ is constant. This is impossible.

Thus $\mathcal{F}$ is holomorphically normal, i.e, $f$ is a normal mapping.

By [JK1, Theorem 2.3], $f$ extends to a holomorphic mapping $f^{*}: \Delta^{n} \rightarrow$ $M$. 
Remark 4.5. (i) By the same argument as the proof of Theorem $\mathrm{C}$ and by [JK1, Theorem 2.3 and Corollary 2.5], we have the following.

Theorem $\mathrm{C}^{\prime}$. Let $M$ be a compact complex space. Let $\mathcal{F} \subset \operatorname{Hol}\left(\left(\Delta^{*}\right)^{n}\right.$, $M)$. Suppose that there exist $q$ hypersurfaces $H_{1}, \ldots, H_{q}$ in $M$ and fixed positive integers $m_{1}, \ldots, m_{q}\left(m_{i} \leq \infty, i=1, \ldots, q\right)$ such that the family $\left\{\left(H_{i}, m_{i}\right)\right\}_{i=1}^{q}$ has the D-property (cf. Definition 4.3) and $f$ intersects $H_{i}$ with multiplicity at least $m_{i}(1 \leq i \leq q)$ for each $f \in \mathcal{F}$.

Then every $f \in \mathcal{F}$ extends to a holomorphic mapping $f^{*}: \Delta^{n} \rightarrow M$ and the family $\mathcal{F}^{*}:=\left\{f^{*}: f \in \mathcal{F}\right\} \subset \operatorname{Hol}\left(\Delta^{n}, M\right)$ is uniformly normal.

(ii) Let $S$ be an analytic subset in $\Omega$. Denote by $\operatorname{Sing}(S)$ the set of singular points of $S$. By Theorem C, $f$ extends to a holomorphic mapping of $\Omega-\operatorname{Sing}(S)$ into $M$. Thus $f$ can be regarded as a meromorphic mapping into $M$. Since $\operatorname{dim} \operatorname{Sing}(S) \leq n-2, f$ extends to a meromorphic mapping in $\Omega$.

We now discuss the problem posed in [Ja, Remark 3]. First of all, we recall the following.

Definition 4.6. Let $\Omega$ be a hyperbolic domain in $\mathbf{C}^{n}$. Let $M$ be a complete Hermitian complex space with a length function $E_{M}$. Let $f \in$ $\operatorname{Hol}(\Omega, M)$. We say that $f$ is a normal holomorphic mapping in the sense of Lehto - Virtanen provided that there exists some positive constant $c$ such that, for all $z \in \Omega$ and all $\xi \in T_{z} \Omega$ it holds that

$$
E_{M}(f(z), d f(z)(\xi)) \leq c F_{K}^{\Omega}(z, \xi)
$$

where $d f(z)$ is the tangent mapping from $T_{z} \Omega$ to $T_{f(z)} M$ induced by $f$ and $F_{K}^{\Omega}$ denotes the infinitesimal Kobayashi metric on $\Omega$.

By $[\mathrm{AK}]$, if $M$ is compact then $f$ is normal if and only if $f$ is normal in the sense of Lehto - Virtanen.

By Lemma 4.2 and Theorem $\mathrm{C}^{\prime}$, we have the partial answer for the above problem of Jarvi.

Corollary 4.7. Let $f:\left(\Delta^{*}\right)^{n} \rightarrow \mathbf{P}^{1}(\mathbf{C})$ be a holomorphic mapping such that the following are satisfied.

(i) There are positive integers $m_{0}, m_{1}, m_{2}\left(m_{i} \leq \infty\right)$ such that $\frac{1}{m_{0}}+$ $\frac{1}{m_{1}}+\frac{1}{m_{2}}<1$. 
(ii) There exist distinct points $a_{0}, a_{1}, a_{2} \in \mathbf{P}^{1}(\mathbf{C})$ such that $f$ has multiplicity at least $m_{i}$ at $a_{i}(0 \leq i \leq 2)$.

Then $f$ extends to a normal holomorphic mapping $f^{*}: \Delta^{n} \rightarrow \mathbf{P}^{1}(\mathbf{C})$.

\section{REFERENCES}

[AK] G. Aladro and S. G. Krantz, A criterion for normality in $\mathbf{C}^{n}$, J. Math. Anal. and App., 161 (1991), 1-8.

[E-S] A. E. Eremenko and M. L. Sodin, The value distribution of meromorphic functions and meromorphic curves from the view point of potential theory, St. Petersburg Math. J., 3 (1992), 109-136.

[Fu1] H. Fujimoto, Extension of the big Picard's theorem, Tohoku Math. J., 24 (1972), $415-422$.

[Fu2] H. Fujimoto, On families of meromorphic maps into the complex projective space, Nagoya Math. J., 54 (1974), 21-51.

[Ja] P. Järvi, An extension theorem for normal functions in several variables, Proc. Amer. Math. Soc., 103 (1988), 1171-1174.

[JK1] J. Joseph and M. H. Kwack, Extension and convergence theorems for families of normal maps in several complex variables, Proc. Amer. Math. Soc., 125 (1997), $1675-1684$.

[JK2] J. Joseph and M. H. Kwack, Some classical theorems and families of normal maps in several complex variables, Complex Variables, 29 (1996), 343-362.

[Ko] S. Kobayashi, Hyperbolic Complex Spaces, Grundlehren der Mathematischen Wissenchaften 318, Springer-Verlag, 1998.

[Kwa] M. H. Kwack, Generalizations of the big Picard theorem, Ann. Math., 90 (1969), 9-22.

[La] S. Lang, Introduction to Complex Hyperbolic Spaces, Springer-Verlag, NY, 1987.

[LeVi] O. Lehto and K. I. Virtanen, Boundary behaviour and normal meromorphic functions, Acta Math., 97 (1957), 47-65.

[Noc] E. Nochka, On the theory of meromorphic functions, Soviet Math. Dokl., 27 (1983), 377-381.

[NO] J. Noguchi and T. Ochiai, Geometric Function Theory in Several Complex Variables, Transl. Math. Monogr. 80, Amer. Math. Soc., 1990.

[No-Wi] J. Noguchi and J. Winkelmann, Holomorphic curves and integral points off divisors, Math. Z., 239 (2002), 593-610.

$[\mathrm{Ru}] \mathrm{M} . \mathrm{Ru}$, Integral points and the hyperbolicity of the complement of hypersurfaces, J. reine angew. Math., 442 (1993), 163-176.

[Rut] H. Rutishauser, Uber die Folgen und Scharen von analytischen und meromorphen Funktionen mehrerer Variabeln, sowie von analytischen Abbildungen, Acta Math., 83 (1950), 249-325.

[S] W. Stoll, Normal families of non-negative divisors, Math. Z., 84 (1964), 154-218. 
[TTH] Do Duc Thai, Pham Nguyen Thu Trang and Pham Dinh Huong, Families of normal maps in several complex variables and hyperbolicity of complex spaces, Complex Variables, 48 (2003), 469-482.

[Tu1] Z.-h Tu, Normality criterions for families of holomorphic mappings of several complex variables into $\mathbf{P}^{N}(\mathbf{C})$, Proc. Amer. Math. Soc., 127 (1999), 1039-1049.

[Tu2] Z.-h Tu, On meromorphically normal families of meromorphic mappings of several complex variables into $\mathbf{P}^{N}(\mathbf{C})$, J. Math. Anal. and App., 267 (2002), 1-19.

[Za] L. Zalcman, Normal families: New perspectives, Bull. Amer. Math. Soc., 35 (1998), 215-230.

Pham Ngoc Mai

Department of Mathematics

Hanoi University of Education

Cau Giay, Hanoi

Vietnam

Do Duc Thai

Department of Mathematics

Hanoi University of Education

Cau Giay, Hanoi

Vietnam

ddthai@netnam. org. vn

Pham Nguyen Thu Trang

Department of Mathematics

Hanoi University of Education

Cau Giay, Hanoi

Vietnam 